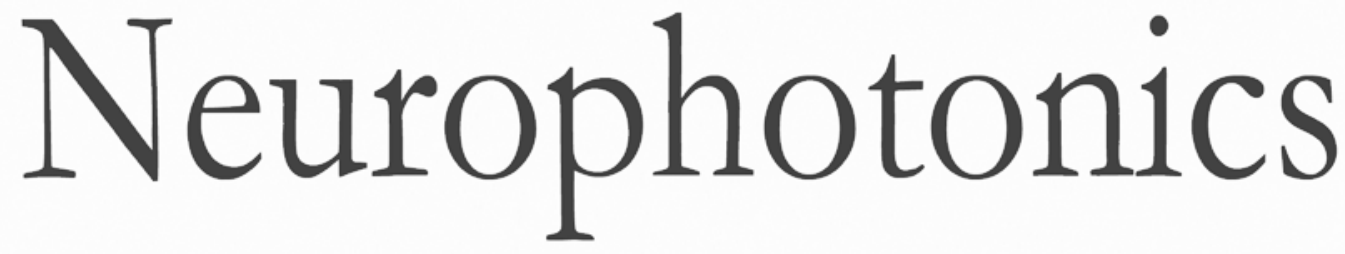

\title{
Prefrontal activity predicts individual differences in optimal attentional strategy for preventing motor performance decline: a functional near-infrared spectroscopy study
}

Takeshi Sakurada

Aya Goto

Masayuki Tetsuka

Takeshi Nakajima

Mitsuya Morita

Shin-ichiroh Yamamoto

Masahiro Hirai

Kensuke Kawai 


\title{
Prefrontal activity predicts individual differences in optimal attentional strategy for preventing motor performance decline: a functional near-infrared spectroscopy study
}

\author{
Takeshi Sakurada, ${ }^{\mathrm{a}, \mathrm{b}, \mathrm{k}, \dagger}$ Aya Goto,,${ }^{\mathrm{a}, \mathrm{c}, \dagger}$ Masayuki Tetsuka, ${ }^{\mathrm{b}}$ Takeshi Nakajima, ${ }^{\mathrm{b}, \mathrm{d}}$ Mitsuya Morita, ${ }^{\mathrm{d}, \mathrm{e}}$ \\ Shin-ichiroh Yamamoto, ${ }^{c}$ Masahiro Hirai, ${ }^{a}$ and Kensuke Kawai ${ }^{b}$ \\ a Jichi Medical University, Center for Development of Advanced Medical Technology, Functional Brain Science Laboratory, Shimotsuke, \\ Tochigi, Japan \\ bJichi Medical University, Department of Neurosurgery, Shimotsuke, Tochigi, Japan \\ 'Shibaura Institute of Technology, College of Systems Engineering and Science, Minuma, Saitama, Japan \\ dJichi Medical University Hospital, Rehabilitation Center, Shimotsuke, Tochigi, Japan \\ e Jichi Medical University, Division of Neurology, Department of Internal Medicine, Shimotsuke, Tochigi, Japan
}

\begin{abstract}
Directing attention to movement outcomes (external focus; EF), not body movements (internal focus; IF), is a better cognitive strategy for motor performance. However, EF is not effective in some healthy individuals or stroke patients. We aimed to identify the neurological basis reflecting the individual optimal attentional strategy using functional near-infrared spectroscopy. Sixty-four participants ( 23 healthy young, 23 healthy elderly, and 18 acute stroke) performed a reaching movement task under IF and EF conditions. Of these, 13 healthy young participants, 11 healthy elderly participants, and 6 stroke patients showed better motor performance under EF conditions (EF-dominant), whereas the others showed IF-dominance. We then measured prefrontal activity during rhythmic hand movements under both attentional conditions. IF-dominant participants showed significantly higher left prefrontal activity than EF-dominant participants under IF condition. In addition, receiver operating characteristic analysis supported that the higher activity in the left frontopolar and dorsolateral prefrontal cortices could detect IF-dominance as an individual's optimal attentional strategy for preventing motor performance decline. Taken together, these results suggest that prefrontal activity during motor tasks reflects an individual's ability to process internal body information, thereby conferring IF-dominance. These findings could be applied for the development of individually optimized rehabilitation programs. $\odot$ The Authors. Published by SPIE under a Creative Commons Attribution 4.0 Unported License. Distribution or reproduction of this work in whole or in part requires full attribution of the original publication, including its DOI. [DOI: 10.1117/1.NPh.6.2.025012]
\end{abstract}

Keywords: focus of attention; individual differences; acute stroke; prefrontal cortex; functional near-infrared spectroscopy; receiver operating characteristic analysis.

Paper 19008RR received Feb. 6, 2019; accepted for publication May 30, 2019; published online Jun. 13, 2019.

\section{Introduction}

Focus of attention is one of the most influential factors facilitating motor performance. ${ }^{1}$ Previous studies have investigated the effects of two distinct attentional strategies on motor performance: internal focus (IF) and external focus (EF). In the IF strategy, performers direct their attention toward body movements, whereas, in the EF strategy, performers direct their attention toward movement outcomes. Most previous studies have found that the EF strategy results in greater improvement in motor performance than the IF strategy in both healthy and clinical populations. ${ }^{2,3}$ The advantage of the EF strategy has been explained by the constrained-action hypothesis, ${ }^{4}$ which states that conscious motor control associated with IF interferes with automatic control processes, whereas directing attention to movement outcomes $(\mathrm{EF})$ reduces such interference. In other words, the EF strategy enhances implicit neural processes for motor control, thereby leading to improved motor performance.

*Address all correspondence to Takeshi Sakurada, E-mail: sakurada@ fc.ritsumei.ac.jp

†These authors contributed equally.
This hypothesis is supported by empirical studies on attentionalcapacity demands, ${ }^{4}$ high-frequency movement adjustments, ${ }^{5}$ and electromyography during motor tasks. ${ }^{6}$

Despite general evidence supporting the EF strategy, several studies have reported that it is not the optimal strategy for all individuals. For example, low-skill golfers showed better performance under IF instructions than EF instructions, ${ }^{7}$ and children who received IF instructions showed more accurate performance in a dart throwing task than those who received EF instructions. ${ }^{8}$ Similarly, a lack of advantage of EF was reported in a legstepping task among some stroke patients. ${ }^{9}$ Our recent studies on healthy and stroke populations demonstrated that the advantage of the EF strategy is dependent on individual modality dominance (visual or kinesthetic) for motor imagery. ${ }^{10,11}$ Although participants with visual imagery dominance showed better motor performance under the EF condition, those with kinesthetic imagery dominance showed higher motor performance under the IF condition. These findings suggest that the optimal combination of attentional strategy and individual motor imagery ability will most improve motor performance.

Although the behavioral evidence for the differential effects of attentional focus strategies on motor performance has accumulated over the past 20 years, the neural basis of this effect 
remains unclear. Indeed, few studies have applied neuroimaging modalities such as functional magnetic resonance imaging during motor tasks to investigate distinct patterns of neural activity under different attentional strategy conditions. In one study that did, a hitting-key task induced greater activity in the primary somatosensory and motor cortices under an EF strategy in which participants attended to keys that needed to be pressed rather than an IF strategy in which participants attended only to finger movement. ${ }^{12}$ Another study reported that neural activation in the left lateral premotor cortex, left primary somatosensory cortex, and intraparietal lobule was induced by the switching of attentional focus during a finger movement task. ${ }^{13}$ Taken together, previous neuroimaging studies strongly suggest that neural activity related to attentional strategy is mainly observed in motor-related areas. However, these studies only explored differences in activity patterns between IF and EF strategiesthey did not consider individual differences in optimal attentional strategy (i.e., differences between EF- and IF-dominant individuals). To the best of our knowledge, there have been no studies on the neural basis of individual differences in optimal attentional strategy.

We hypothesized that the prefrontal cortex is associated with individual differences during motor tasks. There are two reasons for this expectation. First, a large interparticipants variance of motor performance was markedly observed under the IF condition rather than the EF condition. ${ }^{11}$ This trend suggests that the optimal attentional strategy depends mainly on an individual's capacity to process internal body information under the IF condition and not on individual differences in processing external outcome information under the EF condition. From a functional viewpoint, the prefrontal cortex is a well-known critical area for processing internal body information, such as tactile stimuli ${ }^{14}$ and haptic information. ${ }^{15}$ Second, the prefrontal cortex is involved in efficient learning with a limited cognitive capacity, as explained in cognitive load theory. ${ }^{16,17}$ Specifically, previous studies have reported that the prefrontal cortex is related to intrinsic load, which depends on individual skills or task difficulty as well as germane load, which involves the construction of schemas that contribute to learning enhancement. ${ }^{18-20}$ Furthermore, the cognitive ability based on limited capacity, as described above, can change with age and educational background and is reflected as neural activity in the prefrontal cortex. $^{21,22}$ Thus, evaluating prefrontal activity is a reasonable approach for characterizing individual differences in cognitive neural processes. Of note, individual differences in optimal attentional strategy during a motor task have been reported in both healthy and clinical populations. ${ }^{10,11}$ Thus, we expect that the prefrontal cortex is a common neural basis underlying an individual's optimal attentional strategy independent of age or motor disability.

To test our hypotheses, this study examined whether neural activity in the prefrontal area reflects individual optimal attentional strategy for motor performance. If the constrained-action hypothesis is true for all performers of any motor task, we predict that prefrontal activity related to attentional control will not be modulated by individual optimal attentional strategy. Alternatively, if individual cognitive processing capacity mediated by the prefrontal cortex does determine the optimal attentional strategy, we predict that prefrontal activity will be modulated by individual attentional optimality. In this study, we first classified participants into IF- and EF-dominant individuals by comparing motor performance under the IF and EF conditions. Subsequently, based on this classification, we assessed differences in neural activity in the prefrontal area during a simple motor task between IF-dominant and EF-dominant individuals. In addition, to support the commonality of the neural basis for individual optimal attentional strategy, we evaluated the classification accuracy (IF-dominant versus EF-dominant) of differential prefrontal activity in healthy individuals and stroke patients based on receiver operating characteristic (ROC) analysis.

\section{Methods}

\subsection{Participants}

Both clinical and healthy participants were recruited for this study. For the stroke patients, we excluded those with upper limb movement deficits unrelated to stroke as well as those with aphasia, dysarthria, visual field loss, or hemispatial neglect. Because the current task required participants to move their hand while holding a pen, we excluded stroke patients with severe paralysis. Additional exclusion criteria were sensory loss of the upper limb or a minimental state examination (MMSE) ${ }^{23}$ score less than 24. This MMSE cut-off value was also used as an exclusion criterion among the elderly participants. All healthy participants were right-handed, as assessed by the Edinburgh Inventory. ${ }^{24}$

In total, 64 individuals participated in this study: 23 healthy young students recruited from Jichi Medical University (young group), 23 healthy elderly participants with no neurological or skeletomotor dysfunction recruited from the Silver Human Resources Center of Shimotsuke City (elderly group), and 18 patients with acute stroke recruited from the Department of Neurosurgery and Division of Neurology, Department of Medicine, Jichi Medical University (stroke group). We used the Fugl-Meyer Assessment of Motor Recovery (FMA) to assess motor recovery after stroke. ${ }^{25}$ Detailed participant characteristics and the lesion site of each stroke patient are shown in Tables 1 and 2 .

This study was conducted in accordance with the Declaration of Helsinki and approved by the Institutional Review Board at Jichi Medical University. All participants provided written informed consent prior to participation and performed the following two tasks. Each participant completed the two tasks on the same day.

\subsection{Task 1: Individual Optimal Attentional Strategy Classification}

The first task aimed to classify participants as IF-dominant or EF-dominant by evaluating their optimal attentional strategy during a reaching task.

\subsubsection{Experimental setup}

Each participant was seated on a chair facing an LCD monitor. The distance between the participant's eyes and the monitor was $\sim 70 \mathrm{~cm}$. All visual stimuli presented on the monitor were programmed in MATLAB (MathWorks, Natick, Massachusetts) using Cogent Toolbox software (University College London, London, United Kingdom ${ }^{26}$ ). Healthy participants performed the reaching movements using their right hand (task hand). In the stroke group, the unaffected hand was used as the task hand for two patients with moderate hemiplegia (P07 and P10: individual FMA scores are shown in Table 2). Other stroke patients performed the reaching movements with their affected 
Table 1 Participant information.

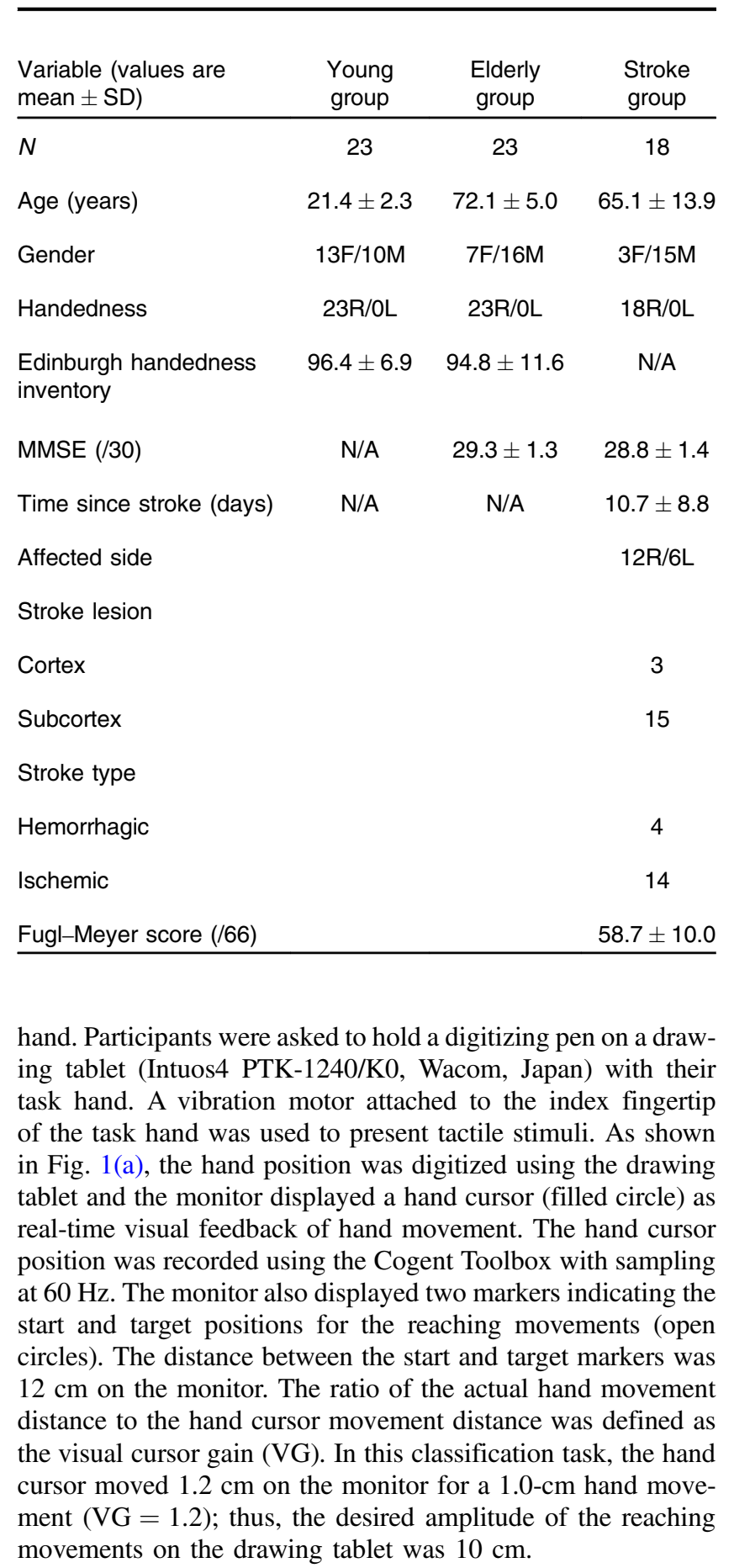

\subsubsection{Procedure}

Figure 1(b) shows the time sequence of a trial. Before starting each trial, the monitor displayed written instructions to remind participants of the attentional strategy to use during the reaching movements. First, participants moved the hand cursor to the start marker when it appeared on the monitor. After a random delay period ( 1 to $2 \mathrm{~s}$ ), a start cue was presented that was either a tactile stimulus delivered by the vibration motor or a brief color change of the hand cursor. The stimulus duration was $0.2 \mathrm{~s}$. Participants were instructed to start the reaching movement as quickly as possible in response to the start cue. Participants
Table 2 Individual FMA scores and lesion sites.

\begin{tabular}{|c|c|c|}
\hline Patient number & FMA score & Localization \\
\hline P01 & 63 & L pons \\
\hline P02 & 50 & $\mathrm{R}$ corona radiata \\
\hline P03 & 57 & $\mathrm{R}$ corona radiata \\
\hline P04 & 63 & L pons \\
\hline P05 & 63 & $\mathrm{~L}$ corona radiata \\
\hline P06 & 66 & $\mathrm{~L}$ corona radiata \\
\hline P07 & 37 & L putamen \\
\hline P08 & 66 & $\mathrm{~L}$ corona radiata \\
\hline P09 & 57 & L putamen \\
\hline P10 & 30 & $L$ thalamus \\
\hline P11 & 63 & $\mathrm{R}$ corona radiata \\
\hline P12 & 63 & L precentral gyrus \\
\hline $\mathrm{P} 13$ & 61 & $\mathrm{~L}$ corona radiata \\
\hline P14 & 64 & $\mathrm{R}$ thalamus \\
\hline P15 & 61 & $L$ thalamus \\
\hline P16 & 64 & $\mathrm{R}$ precentral gyrus \\
\hline P17 & 66 & $\mathrm{R}$ precentral gyrus \\
\hline P18 & 62 & $L$ thalamus \\
\hline
\end{tabular}

were also instructed to control the hand cursor in as straight a line as possible from the start marker to the target marker. The trial ended $1 \mathrm{~s}$ after reaching the target marker.

We then introduced the two experimental conditions, namely IF and EF. Under the IF condition, because participants were instructed to covertly direct attention to their hand movements, trials with a tactile start cue and those with a visual start cue were defined as consistent-trials and inconsistent-trials, respectively. By contrast, under the EF condition, participants were instructed to direct their attention only to the hand cursor on the monitor. Therefore, trials beginning with the visual start cue were defined as consistent-trials and those with the tactile start cue were defined as inconsistent-trials.

The classification task comprised two sessions. The IF and EF conditions were randomly assigned for the first and second sessions for each participant. Each session had three phases: baseline, learning, and relearning.

\section{- Baseline phase.}

The hand cursor moved contingently according to the participant's hand movement (visuomotor rotation angle $=0 \mathrm{deg}$ ). All participants performed nine consistent-trials.

- Learning phase.

Clockwise $(\mathrm{CW})$ or counterclockwise $(\mathrm{CCW})$ visuomotor rotation was applied to the hand cursor movements. In 
(a)

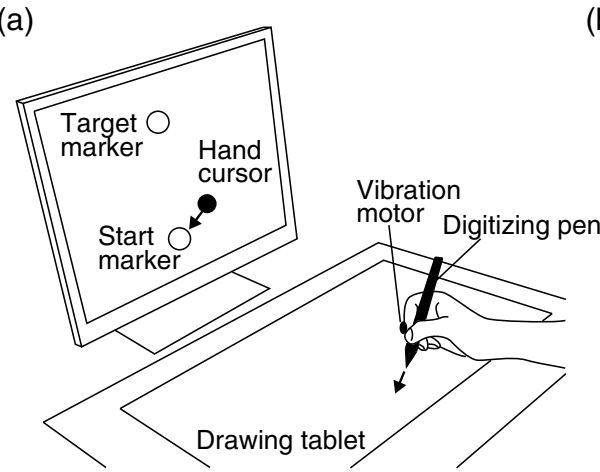

(b)

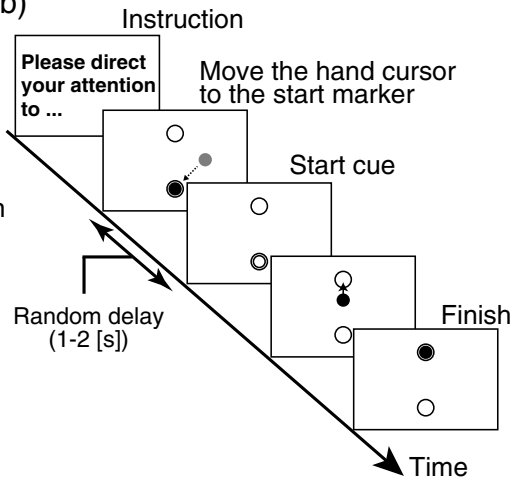

Fig. 1 Experimental design. (a) Experimental setup. During performing the classification tasks, the participant's task hand was occluded by a small rack. Thus, the participant could not see their hand while they performed the experimental tasks. The positions of the start and target markers were the same in all trials. (b) Time sequence of one trial. This case indicates the EF condition with visual start cue.

visuomotor rotation trials, hand cursor movement was rotated by 45 deg from the origin (start marker) in either the CW or CCW direction relative to the participant's actual hand movement. Participants were required to correctly modify their hand movements to control the hand cursor in a straight trajectory. The $\mathrm{CW}$ and $\mathrm{CCW}$ settings were randomly assigned to the IF and EF conditions. Half of participants performed the reaching movements under the IF condition with the $\mathrm{CW}$ rotation and the EF condition with the CCW rotation, whereas the other participants performed the reaching movements under the IF condition with the $\mathrm{CCW}$ rotation and the $\mathrm{EF}$ condition with the $\mathrm{CW}$ rotation.

The young and elderly groups performed 40 trials (35 consistent-trials and 5 inconsistent-trials) while the stroke group performed 30 trials to avoid fatigue (27 consistent-trials and 3 inconsistent-trials). The inconsistent-trials were randomly inserted among the consistent-trials. The baseline and learning phase trials were run without interruption and participants were not informed when $\mathrm{CW}$ or $\mathrm{CCW}$ rotation started.

\section{- Relearning phase.}

There was a 10-min break after the learning phase. All participants then performed nine consistent-trials again as the relearning phase. The visual feedback settings on the monitor, including the rotation angle, were identical to those in the learning phase.

\subsubsection{Analysis}

Reaction time. To confirm whether participants correctly directed their attention according to the experimental instructions, we applied the same method as used in our previous study. ${ }^{10}$ In the learning phase, we analyzed the reaction time (RT) of each trial, defined as the delay from the presentation of a start cue (tactile or visual) to the instant where tangential hand velocity exceeds $50 \mathrm{~mm} / \mathrm{s}$. For further analysis, we excluded RTs faster than $150 \mathrm{~ms}$ or slower than $1500 \mathrm{~ms}^{27,28}$ Participants were considered to correctly direct their attention when the mean RT satisfied one of the following two equations:

$\left(\mathrm{RT}_{\mathrm{IF}}^{\mathrm{T}}<\mathrm{RT}_{\mathrm{EF}}^{\mathrm{T}}\right) \cap\left(\mathrm{RT}_{\mathrm{EF}}^{\mathrm{V}}<\mathrm{RT}_{\mathrm{IF}}^{\mathrm{V}}\right)$

$\left(\mathrm{RT}_{\mathrm{IF}}^{\mathrm{T}}<\mathrm{RT}_{\mathrm{IF}}^{\mathrm{V}}\right) \cap\left(\mathrm{RT}_{\mathrm{EF}}^{\mathrm{V}}<\mathrm{RT}_{\mathrm{EF}}^{\mathrm{T}}\right)$.
Here, the superscripts $\mathrm{T}$ and $\mathrm{V}$ denote the modality of the start cue (tactile: T, visual: V), and the subscripts IF and EF denote the attentional condition. Equations (1) and (2) both represent the situation where RT is faster in consistent-trials than in inconsistent-trials.

Motor performance. In each trial, we quantified movement error as the area enclosed by the hand trajectory and a straight line connecting the start and target markers (in $\mathrm{cm}^{2}$ ). Once motor skills are acquired, they can be retained over extended periods. ${ }^{29}$ Therefore, to classify participants into IF-dominant and EF-dominant individuals, we calculated the degree of motor performance decline after a short break between the learning and relearning phases. Namely, we subtracted the mean movement error of the last three trials in the learning phase from that of the first three trials in the relearning phase in each attentional condition $\left(D_{\mathrm{IF}}\right.$ and $\left.D_{\mathrm{EF}}\right)$. Next, we individually subtracted the degree of motor performance decline under the IF condition from that under the $\mathrm{EF}$ condition $\left(\Delta D=D_{\mathrm{EF}}-D_{\mathrm{IF}}\right)$. We defined the IF-dominant subgroup as participants who showed a smaller motor performance decline under the IF condition than the EF condition $(\Delta D>0)$, and the EF-dominant subgroup as participants who showed a smaller motor performance decline under the EF condition than the IF condition $(\Delta D<0)$.

\subsubsection{Statistical analysis}

Differences in RT were assessed by three-way repeated measures analysis of variance (ANOVA) with group (young, elderly, or stroke) as a between-subjects factor and condition (IF condition or EF condition) and modality of the start cue (tactile or visual stimuli) as within-subject factors. To evaluate the degree of motor performance decline, a three-way ANOVA was also applied to the motor performance decline with group (young, elderly, or stroke) and individual dominance (IF-dominant or EF-dominant) as between-subjects factors and condition (IF condition or EF condition) as a within-subject factor. We used a significance threshold of $p<0.05$ (two-tailed) for all tests.

\subsection{Task 2: Recording Prefrontal Activity Using fNIRS}

The second task aimed to identify the common neural basis underlying individual differences in optimal attentional strategy 
among healthy and clinical populations. For this purpose, we compared prefrontal activity between IF-dominant and EFdominant individuals based on the results of the first classification task.

\subsubsection{Experimental setup}

For behavioral data acquisition, we used the same experimental setup as in the first classification task (i.e., we recorded task hand movements using a drawing tablet with a monitor displaying a hand cursor as real-time visual feedback). To record prefrontal activity, we used a multichannel fNIRS system (ETG-7100, Hitachi Medical Corporation, Kashiwa, Japan) with sampling at $10 \mathrm{~Hz}$. The fNIRS probes were arranged to cover the prefrontal area [Fig. 2(a)]. We used a $3 \times 5$ multichannel probe holder consisting of eight laser sources emitting at 695 and $830 \mathrm{~nm}$ [emitters; red squares in Fig. 2(a)] and seven detecting probes [detectors; blue squares in Fig. 2(a)] arranged alternately at an interprobe distance of $3 \mathrm{~cm}$. The midpoint of an emitter/ detector pair was defined as a recording channel location [circles in Fig. 2(a)]. The probe holder was placed on the scalp with its lowest-row center emitter at the participant's Fpz position, according to the standard international 10-20 system.

fNIRS signals reflect hemoglobin changes originating in cortical tissue due to brain activation and skin blood flow. To eliminate the influence of skin blood flow on fNIRS signals, we set eight additional short detecting probes at an interprobe distance of $1.5 \mathrm{~cm}$ [light blue squares in Fig. 2(a)] and applied multidistance independent component analysis (ICA) ${ }^{30}$ ICA is a signal discrimination method in which independent components are extracted from mixed signals. It has been successfully used for fNIRS analysis. ${ }^{31-34}$ Because signals from recording channels with a $1.5-\mathrm{cm}$ interprobe distance primarily include skin blood flow signals in shallow tissues, based on these signals, we could discriminate between the effects of cortical tissue and skin blood flow on fNIRS signals. As it was possible to apply multidistance ICA only to the recording channels around the short detecting probes, the number of available recording channels was reduced to 15 [numbered circles in Fig. 2(a)]. For the spatial registration of fNIRS maps onto Montreal Neurological

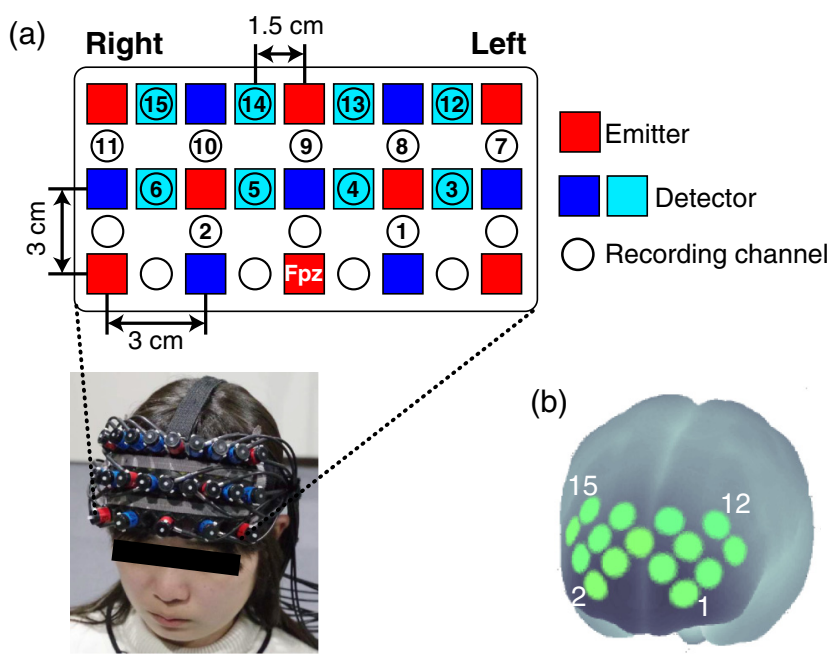

Fig. 2 Probe configuration for near-infrared spectroscopy. (a) Probes were placed over the prefrontal area. (b) Spatial registration of fNIRS maps onto MNI coordinate space. Each recording channel is numbered from the lower left to upper right position.
Institute (MNI) coordinate space, we measured scalp landmarks and all fNIRS recording channel positions using a threedimensional magnetic space digitizer (FASTRAK, Polhemus). We then used an estimation tool without MRI. ${ }^{35}$ Details on the spatial profiling of recording channels are provided in Fig. 2(b) and Table 3 .

Table 3 Spatial profiling of each recording channel.

\begin{tabular}{|c|c|c|c|}
\hline ch. & Localization & Brodmann area & Probability \\
\hline 1 & Left FPC & 10 & 1 \\
\hline 2 & Right FPC & 10 & 1 \\
\hline 3 & Left FPC & 10 & 1 \\
\hline 4 & Left FPC & 10 & 1 \\
\hline 5 & Right FPC & 10 & 1 \\
\hline \multirow[t]{2}{*}{6} & Right FPC & 10 & 0.97 \\
\hline & Right DLPFC & 46 & 0.03 \\
\hline \multirow[t]{3}{*}{7} & Left DLPFC & 46 & 0.72 \\
\hline & Left FPC & 10 & 0.21 \\
\hline & Left DLPFC & 9 & 0.07 \\
\hline \multirow[t]{2}{*}{8} & Left FPC & 10 & 0.57 \\
\hline & Left DLPFC & 9 & 0.43 \\
\hline \multirow[t]{2}{*}{9} & Right FPC & 10 & 0.66 \\
\hline & Right DLPFC & 9 & 0.34 \\
\hline \multirow[t]{2}{*}{10} & Right FPC & 10 & 0.55 \\
\hline & Right DLPFC & 9 & 0.45 \\
\hline \multirow[t]{3}{*}{11} & Right DLPFC & 46 & 0.86 \\
\hline & Right FPC & 10 & 0.12 \\
\hline & Right DLPFC & 9 & 0.02 \\
\hline \multirow[t]{3}{*}{12} & Left DLPFC & 9 & 0.71 \\
\hline & Left includes FEF & 8 & 0.27 \\
\hline & Left DLPFC & 46 & 0.02 \\
\hline \multirow[t]{2}{*}{13} & Left DLPFC & 9 & 0.64 \\
\hline & Left includes FEF & 8 & 0.36 \\
\hline \multirow[t]{2}{*}{14} & Right DLPFC & 9 & 0.61 \\
\hline & Right includes FEF & 8 & 0.39 \\
\hline \multirow[t]{3}{*}{15} & Right DLPFC & 9 & 0.73 \\
\hline & Right includes FEF & 8 & 0.22 \\
\hline & Right DLPFC & 46 & 0.05 \\
\hline
\end{tabular}

Note: FPC, frontopolar cortex; DLPFC, dorsolateral prefrontal cortex; FEF, frontal eye fields. 


\subsubsection{Procedure}

The fNIRS task consisted of two sessions each comprising six block sets containing alternating rest (20 s) and motor (15 s) blocks. A rest block was inserted at the end of each session. To avoid fatigue in stroke patients during successive hand movements, we applied a block design with relatively longer rest blocks than motor blocks. As in the first classification task, we introduced two attentional conditions and assigned the IF and EF conditions randomly to first and second sessions for each participant.

\section{- Rest block}

The monitor displayed the rest marker and hand cursor during all rest blocks. Regardless of the attentional condition, participants were required to match the hand cursor to the rest marker at the center of the monitor [Fig. 3(a)].

\section{- Motor block.}

To isolate the habituation effect of the longitudinal direction movements applied in the first classification task, we required the participants to perform cyclic movements in a lateral direction in the second fNIRS task. When each motor block started, the rest marker disappeared and participants were asked to move their hand cyclically on the drawing tablet from side to side at a comfortable speed. The hand cursor on the monitor moved horizontally according to the rightward or leftward hand movements.

Under the IF condition, participants were instructed to covertly direct their attention to their hand movements. To correctly guide participants' attention during the motor blocks, we prepared additional stimuli for cueing the turning point (direction change) of cyclic movements. If a participant's hand position exceeded $\pm 10 \mathrm{~cm}$ in the $x$-axial direction from the center of the drawing tablet, tactile stimuli were provided on the index fingertip by the vibration motor. Participants were requested to keep their hand movement amplitude constant throughout the motor block based on presentation of tactile stimuli (i.e., the desired hand movement amplitude was maintained at $20 \mathrm{~cm}$ on the drawing tablet). Furthermore, to confirm participants' attentional strategies, VG was gradually decreased to from 1 to 0.6. If a participant correctly maintained the desired hand movement amplitude, the hand cursor movement amplitude on the monitor gradually decreased from 20 to $12 \mathrm{~cm}$ [Fig. 3(b)].

By contrast, under the EF condition, participants were instructed to direct their attention only to the hand cursor on the monitor. To indicate the turning point, the color of the hand cursor changed when the hand cursor position exceeded $\pm 10 \mathrm{~cm}$ in the $x$-axial direction from the monitor center. Participants were also requested to keep the hand cursor movement amplitude constant throughout the motor block based on presentation of the visual stimuli (i.e., the desired hand cursor amplitude was $20 \mathrm{~cm}$ on the monitor). VG gradually increased from 1 to 1.5 , so the amplitude of hand movement on the drawing tablet was expected to gradually decrease from 20 to $12 \mathrm{~cm}$ [Fig. 3(c)].

\subsubsection{Analysis}

Movement amplitude. The movement amplitudes of the hand and hand cursor in each cycle were calculated from the difference between the positional peaks along the $x$-axis. The profiles in Figs. 3(b) and 3(c) show typical behavioral results of one participant. By subtracting the amplitude of the first cycle from that

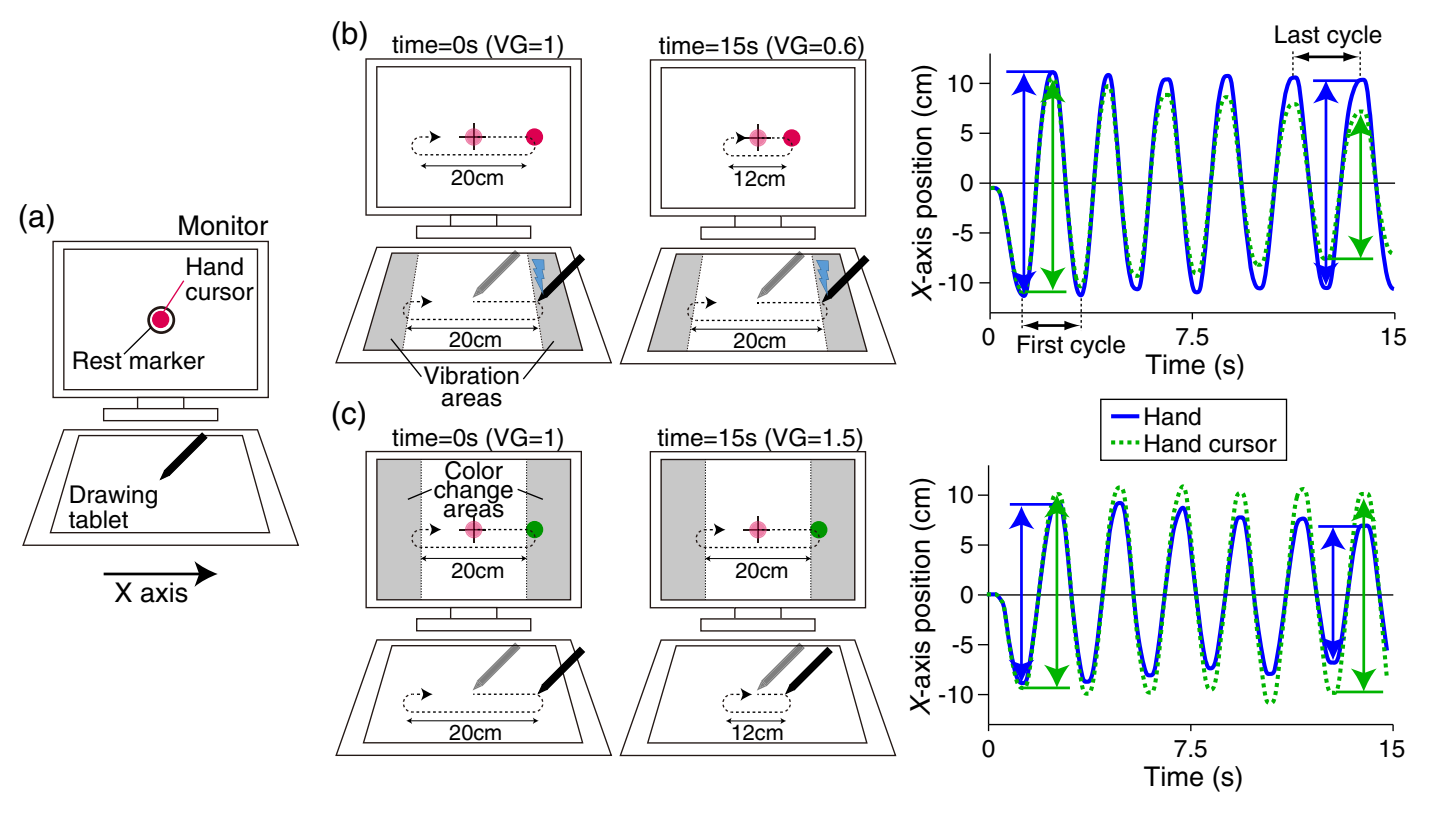

Fig. 3 Task configurations in the fNIRS task. (a) Visual settings in the rest block. (b) VG setting under the IF condition. In the motor block, VG gradually decreased over time. If the participant correctly directed attention to their hand according to the task instructions, the decreasing amplitude was anticipated only in hand cursor movement, as shown by the movement profiles in the right column. The amplitudes of the first and last cycles were defined by the difference between adjacent positional peaks. Blue and green arrows indicate hand and hand cursor amplitudes, respectively. The differential value between the first and last amplitudes was then calculated. (c) VG setting under the EF condition. VG gradually increased over time. If the participant correctly directed their attention to the hand cursor according to the task instructions, the decreasing amplitude was anticipated only in hand movement. 
of the last cycle in each motor block, we calculated the absolute amplitude changes of hand movement $\left(\Delta \mathrm{Amp}_{\mathrm{H}}\right)$ and hand cursor movement $\left(\Delta \mathrm{Amp}_{\mathrm{C}}\right)$. When a participant successfully kept the hand amplitude constant under the IF condition (i.e., $\Delta \mathrm{Amp}_{\mathrm{H}} \ll \Delta \mathrm{Amp}_{\mathrm{C}}$ ) or kept the amplitude of the hand cursor constant under the $\mathrm{EF}$ condition (i.e., $\Delta \mathrm{Amp}_{\mathrm{C}} \ll \Delta \mathrm{Amp}_{\mathrm{H}}$ ), we considered that participant to have correctly directed their attention according to the experimental instructions.

Preprocessing for fNIRS data. To estimate local neural activity, we measured oxygenated hemoglobin (oxy-Hb) and deoxygenated hemoglobin (deoxy-Hb) signals. To remove baseline drift, individual time course data for the oxy- $\mathrm{Hb}$ and deoxy-Hb signals of each channel were high-pass filtered using a cut-off frequency of $0.0167 \mathrm{~Hz}$. Next, to remove blocks with motion-related artifacts, we applied an artifact detection algorithm based on the HOMER2 software for the oxy-Hb and deoxy-Hb signals (MGH-Martinos Center for Biomedical Imaging ${ }^{36}$ ). As no blocks with artifacts were detected, we analyzed all time course data of the oxy-Hb and deoxy-Hb signals.

General linear model (GLM) analysis ${ }^{37,38}$ for prefrontal activity. Previous studies have reported that GLM is capable of detecting task-related hemodynamic changes in the cortex in fNIRS data. ${ }^{39,40}$ In this study, to identify prefrontal regions related to individual differences in optimal attentional strategy, we used GLM analysis with least-squares estimation of the oxy-Hb and deoxy-Hb signals. For the preprocessed oxy-Hb and deoxy-Hb signals, a Gaussian function with a peak time of $6 \mathrm{~s}$ and full-width half-maximum of $5.4 \mathrm{~s}$ were used as a hemodynamic response function to better mimic brain signals. The resulting beta values at each recording channel estimated by the GLM analysis were then used in the group analysis to determine the experimental task effect.

Prefrontal activity-based classification. We tried to classify the optimal attentional strategy using the estimated beta values reflecting individual prefrontal activity and to evaluate the classification accuracy using leave-one-out cross-validation. To select the channels reflecting the optimal attentional strategy, we focused on the significance of the inter-subgroup difference between IF- and EF-dominant individuals. Next, to calculate classification accuracy, a classification index (CI) from a weighted linear summation model was calculated for each participant using

$\mathrm{CI}=\sum_{\mathrm{ch}}\left(k_{\mathrm{ch}} \times \beta_{\mathrm{ch}}\right)$,

where the subscript ch denotes the selected channel number and $k$ is a weighted coefficient of the selected channel. Based on CI excluding one participant, we searched for the best cut-off value to distinguish IF-dominant from EF-dominant individuals. For this purpose, an ROC analysis was performed. The sensitivity (i.e., true-positive rate) was plotted against the $1-$ specificity (i.e., false-positive rate) and accuracy was measured as the area under the curve (AUC). To determine the best weighted coefficients for this prefrontal activity-based classification, these steps were repeated while changing $k$ from 0 to 1 in 0.01 increments to identify the value yielding the highest AUC. It should be noted that when the number of channels with a significant inter-subgroup difference is $1, \mathrm{CI}$ is identical to the beta value in each participant (i.e., $k=1$ ). For the excluded participant, we determined whether an IF- or EF-dominant individual was above or below a given cut-off value for CI. Next, to quantify the classification accuracy, we replaced the excluded participant, recalculated the cut-off value, and classified the excluded participant's optimal attentional strategy (repeated 64 times in total).

\subsubsection{Statistical analysis}

Movement amplitudes were analyzed by three-way repeated measures ANOVA with group (young, elderly, or stroke) as a between-subjects factor and condition (IF or EF) and attentional target (hand or hand cursor) as within-subject factors. To explore intersubgroup differences in the prefrontal activity, we compared the beta values of oxy-Hb and deoxy-Hb signals between the IF- and EF-dominant subgroups in each recording channel using $t$-test. To adjust for multiple testing, we applied false discovery rate (FDR) correction ${ }^{41,42}$ with the significance threshold set at $p<0.05$ (FDR-corrected). Regarding the AUC, because the sample size is insufficient for classification analysis, to alternatively assess the original highest AUC, we repeatedly calculated AUCs on the basis of a randomized group of participants (i.e., we randomly classified the participants into IF- and EFdominant subgroups and calculated AUC 3000 times). Then, we compared the original highest AUC and the randomized data-based AUCs.

\section{Results}

\subsection{Task 1: Individual Optimal Attentional Strategy Classification}

\subsubsection{Reaction time}

We excluded trials in which RT did not meet our criterion $(<150$ or $>1500 \mathrm{~ms}$ ). In total, $3.0 \%$ of tactile-cued and $2.1 \%$ of visualcued responses were excluded under the IF condition, whereas $4.3 \%$ of tactile-cued and $1.9 \%$ of visual-cued responses were excluded under the EF condition.

Comparison of RTs revealed a significant two-way interaction of condition $\times$ modality $[F(1,61)=226.73, p<$ $\left.0.000001, \eta_{p}^{2}=0.79\right]$ and main effects of group $[F(2,61)=$ $\left.12.44, p=000029, \eta_{p}^{2}=0.29\right]$, condition $[F(1,61)=11.05$, $\left.p=0.0015, \quad \eta_{p}^{2}=0.15\right], \quad$ and modality $[F(1,61)=9.04$, $\left.p=0.0038, \eta_{p}^{2}=0.13\right]$. Post-hoc analysis with Bonferroni correction for the condition $\times$ modality interaction revealed that RTs for consistent-trials [tactile in the IF condition: $519.8 \pm$ 16.5 (SEM) $\mathrm{ms}$, visual in the EF condition: $517.2 \pm 15.2$ (SEM) ms] were significantly faster than those for inconsistent-trials [tactile in the EF condition: $617.9 \pm 18.1$ (SEM) ms, visual in the IF condition: $678.2 \pm 20.8(\mathrm{SEM}) \mathrm{ms}, p<1.1 \times$ $\left.10^{-10}\right]$, which is consistent with the requirements of Eq. (1) or Eq. (2) and suggests that participants correctly directed their attention according to condition (to their hand in IF trials or the hand cursor in EF trials).

\subsubsection{Motor performance and individual optimal attentional strategy}

Figures 4(a)-4(c) show the changes in movement errors as the consistent-trials progressed [young, Fig. 4(a); elderly, Fig. 4(b); stroke, Fig. 4(c)]. All groups showed the same trend. In the baseline phase without visuomotor rotation, the hand cursor did not 
(a)

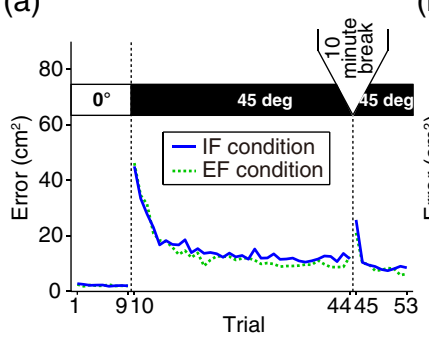

(c)

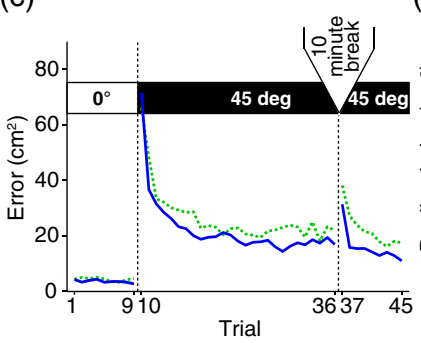

(b)

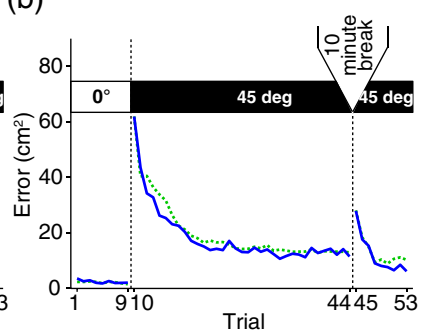

(d)

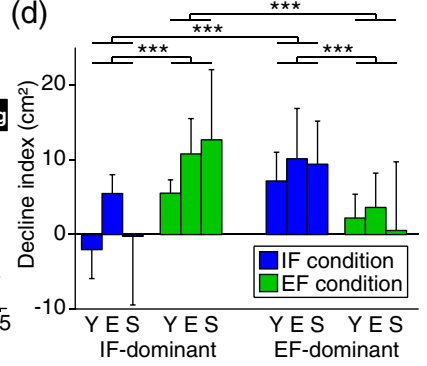

Fig. 4 Learning curve in each group. (a)-(c) Movement errors in the (a) young, (b) elderly, and (c) stroke groups. Blue solid and green dotted lines indicate mean movement errors under the IF and EF conditions, respectively. In all groups, performance improved as trials progressed in the learning phase. After the 10-min break, the error increased and participants readapted to the same visuomotor rotation environment. (d) The degree of motor performance decline under IF and EF conditions. Labels "Y," "E," and "S" indicate the young, elderly, and stroke groups, respectively. Error bars denote the standard deviation. ${ }^{* * *} p<0.001$.

deviate from the required straight trajectory. When the hand cursor movement was rotated relative to the actual movement during the learning phase (starting after the 10th trial), movement error markedly increased and then gradually decreased. Toward the end of the learning phase, the error reached a plateau, indicating that participants had adapted to the visuomotor rotation. After the 10 min break, regardless of the same visuomotor rotation disturbing hand movement in the relearning phase, participants quickly readapted.

By comparing the degree of motor performance decline between the IF and EF conditions as shown in Fig. 4(d), we classified participants into an IF-dominant subgroup $(\Delta D>0$, young: $n=10$, elderly: $n=12$, stroke: $n=12$ ) and EFdominant subgroup $(\Delta D<0$, young: $n=13$, elderly: $n=11$, stroke: $n=6)$. Analysis of motor performance decline revealed a significant main effect of group $[F(2,58)=4.96, p=0.010$, $\left.\eta_{p}^{2}=0.15\right]$ and a significant two-way interaction of condition $\times$ individual dominance $\left[F(1,58)=70.04, \quad p=1.48 \times 10^{-11}\right.$, $\left.\eta_{p}^{2}=0.55\right]$. The other factors did not reach the level of statistical significance $(F \mathrm{~s}<2.73, p \mathrm{~s}>0.074)$. Post-hoc analysis with Bonferroni correction found that motor performance decline in the elderly group was significantly larger than that in young group ( $p=0.011)$. Furthermore, Bonferroni correction revealed that motor performance declines under the IF condition were significantly smaller than those under the EF condition in the IF-dominant subgroup $\left(p=1.20 \times 10^{-8}\right)$. Conversely, the declines under the EF condition were significantly smaller than those under the IF condition in the EF-dominant subgroup $\left(p=5.46 \times 10^{-5}\right)$. The intersubgroup differences under the same attentional condition also reached significance in both conditions (IF condition: $p=8.14 \times 10^{-6}$, EF condition: $\left.p=6.76 \times 10^{-6}\right)$.

\subsection{Task 2: Recording Prefrontal Activity Using fNIRS}

\subsubsection{Movement amplitude}

Analysis of amplitude change revealed a significant three-way interaction of group $\times$ condition $\times$ attentional target $[F(2,61)=$ $\left.3.57, p=0.034, \eta_{p}^{2}=0.10\right]$. Further, we confirmed two important aspects of this three-way interaction by multiple comparison using Bonferroni correction. First, the amplitude changes of attentional target $\left\{\Delta \mathrm{Amp}_{\mathrm{H}}\right.$ under the IF condition $[8.89 \pm$ $1.57(\mathrm{SEM}) \mathrm{cm}]$ and $\Delta \mathrm{Amp}_{\mathrm{C}}$ under the EF condition $[7.91 \pm$ $1.32(\mathrm{SEM}) \mathrm{cm}]\}$ were significantly smaller than those of the nonattentional target $\left\{\Delta \mathrm{Amp}_{\mathrm{C}}\right.$ under the IF condition $[26.4 \pm$ $3.01(\mathrm{SEM}) \mathrm{cm}]$ and $\Delta \mathrm{Amp}_{\mathrm{H}}$ under the $\mathrm{EF}$ condition $[31.2 \pm$ $3.79(\mathrm{SEM}) \mathrm{cm}]\}$, respectively (all $p \mathrm{~s}<0.0088$ ). Therefore, we can assume that participants correctly directed their attention to their hand in the IF condition or the hand cursor in the EF condition according to the experimental instructions. Second, the amplitude changes of the hand and hand cursor did not show any significant differences among the young, elderly, and stroke groups (all $p \mathrm{~s}=0.99$ ). These results indicate that prefrontal activity was not affected by individual motor performance itself.

\subsubsection{Prefrontal activity}

Based on the individual optimal attentional strategy evaluated in the first classification task, we compared the prefrontal activities between the IF-dominant subgroup $(n=34)$ and EF-dominant subgroup $(n=30)$.

Figures 5(a) and 5(b) present the temporal mean profiles of oxy-Hb signals (upper rows) and deoxy-Hb signals (lower rows) across the motor blocks of the same attentional condition in the (a) IF-dominant and (b) EF-dominant subgroups, respectively. The profiles in Fig. 5 show oxy-/deoxy-Hb signals that mainly reflect the effect of the cortical tissue (i.e., the signals after removing the component of the skin blood flow using multidistance ICA). Several channels in both subgroups showed similar trends. First, oxy-Hb signals increased whereas deoxy-Hb signals decreased in several areas. Second, changes in oxy-Hb signals were stronger than those in deoxy-Hb signals. These characteristics are typical hemoglobin dynamics reflecting neural activity. Notably, for oxy-Hb signal under the IF condition, we observed a marked intersubgroup difference in the boundary area including the left DLPFC and left frontopolar cortex (FPC [ch.8: squares in Figs. 5(a) and 5(b)]. More specifically, IF-dominant individuals showed a gradual increase in oxy-Hb signal during performing the cyclic motor task, whereas EF-dominant individuals did not show oxy-Hb signal changes [Fig. 5(c)]. As shown in Fig. 5(d), compared with EF-dominant individuals, IF-dominant individuals showed stronger oxy-Hb signal, especially after the latter half of the motor block.

Figure 6(a) shows the spatial profiles of the $t$-values of the $t$-test between the IF- and EF-dominant subgroups for each recording channel's beta value. Only oxy-Hb signal in the boundary area including the left DLPFC and left FPC (8ch) showed a significant intersubgroup difference under the IF condition [Fig. 6(a)]. In this channel, the IF-dominant subgroup showed higher beta values of oxy-Hb signal than the EF-dominant subgroup [Fig. 6(b) ( $p=0.0016$, FDR-corrected)]. Incidentally, weak intersubgroup differences of oxy-Hb signals were observed in the left FPC [ch.4 under the IF condition $(p=0.012$, 

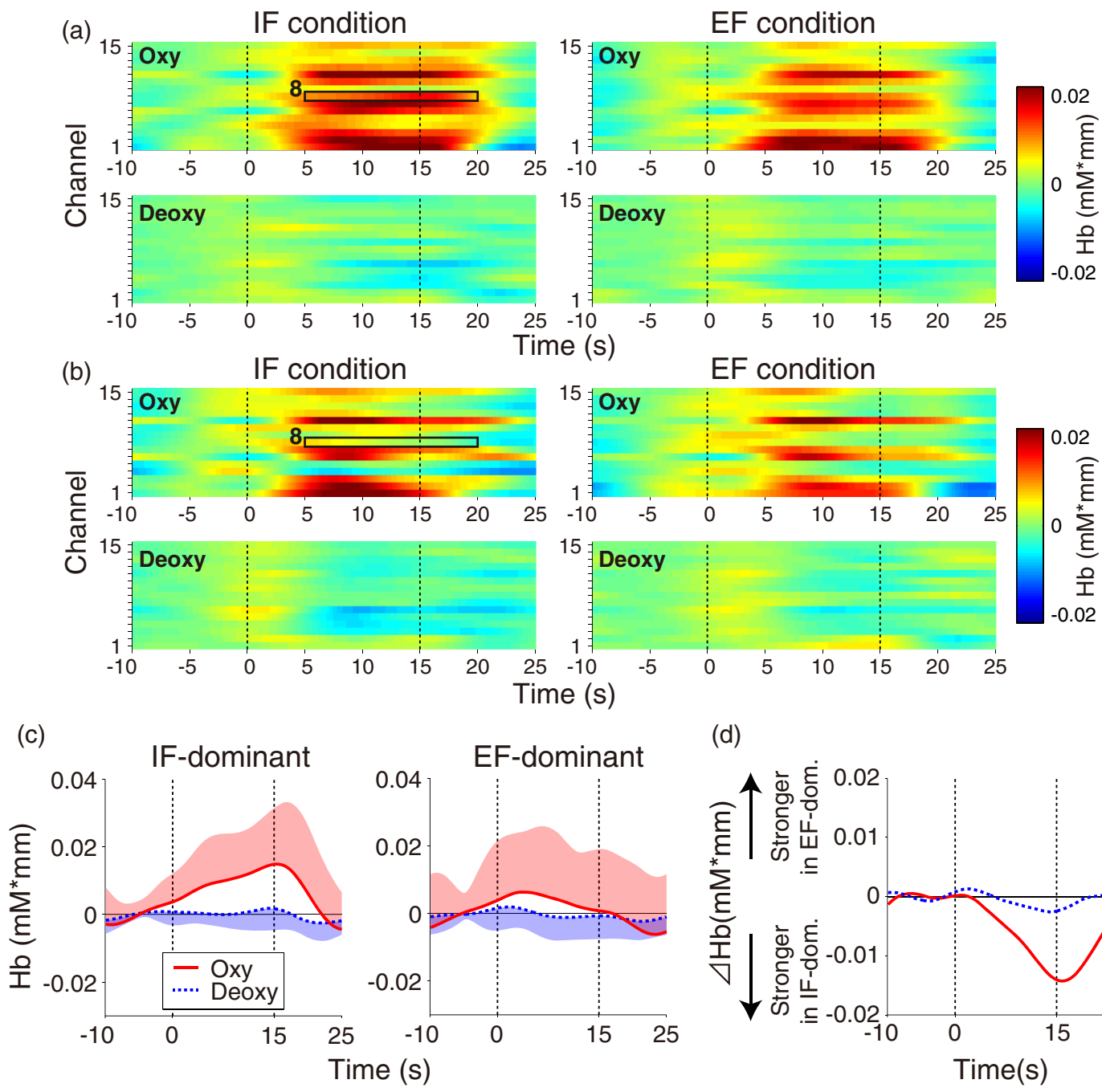

(d)

Fig. 5 Temporal characteristics of oxy- $\mathrm{Hb}$ and deoxy- $\mathrm{Hb}$ signals. Zero on the horizontal axis indicates the start timing of the motor block. (a, b) Upper row: Under the IF condition, oxy-Hb signal in ch. 8 showed a marked difference between the IF- and EF-dominant subgroups. Lower row: Deoxy-Hb signals showed neither strong temporal change nor intersubgroup difference. (c) Temporal profiles of oxy- $\mathrm{Hb}$ and deoxy$\mathrm{Hb}$ signals in ch.8 under the IF condition. Red solid and blue dotted lines represent the time courses of $\mathrm{oxy}-\mathrm{Hb}$ and deoxy-Hb signals, respectively. The lighter colored regions around the time course data indicate the standard deviation. Note that the profiles of oxy- $\mathrm{Hb}$ and deoxy- $\mathrm{Hb}$ signals show only upper red and lower blue standard deviation regions, respectively. (d) The differential mean profiles between the IF- and EF-dominant subgroups (EF-dominant subgroup minus IF-dominant subgroup). Positive values on the longitudinal axis indicate a stronger oxy-/deoxy-Hb signal in the EF-dominant subgroup than in the IF-dominant subgroup, whereas negative values indicate the opposite magnitude relation.

uncorrected)], in the boundary area including the left DFLPC and left FPC [ch.8 under the EF condition ( $p=0.0063$, uncorrected)], and in the left DLPFC [ch.12 under the IF condition ( $p=0.020$, uncorrected) and EF condition ( $p=0.024$, uncorrected)]. Regarding deoxy-Hb signals, we observed weak intersubgroup difference only in the boundary area including the right DFLPC and right FPC [ch.10 under the EF condition $(p=0.039$, uncorrected)] and the left DLPFC [ch.13 under the IF condition ( $p=0.037$, uncorrected)].

\subsubsection{Prefrontal activity-based classification}

Using the estimated beta values of oxy-Hb signal in ch.8 under the IF condition, we then attempted to predict an individual's optimal attentional strategy. Because the number of channels with a significant intersubgroup difference was 1, we applied the individual's beta value as the CI. In the repeated ROC analysis based on leave-one-out cross-validation, the highest AUC was 0.74 (Fig. 7) and the mean AUC was $0.74 \pm 0.00027$ SD. At the optimal cut-off value, IF- and EF-dominant individuals were distinguished with a sensitivity of 0.71 and specificity of 0.73 (open circle in Fig. 7). In addition, we confirmed that the original highest AUC was relatively higher than the randomized data-based AUCs (AUC range: 0.729 to 0.500 ; ratio of 0.7 or more: $0.37 \%$ ). Furthermore, the classification accuracy using the optimal cut-off value was $71.9 \%$ (46 of 64 for all participants). The classification accuracies in each group were $73.9 \%$ [young group: 17 of 23 (females, 9 of 13; males, 8 of 10)], 69.6\% [elderly group: 16 of 23 (females, 6 of 7 ; males, 10 of 16)], and $72.2 \%$ [stroke group: 13 of 18 (females, 3 of 3; males, 10 of 15)]. 
(a)

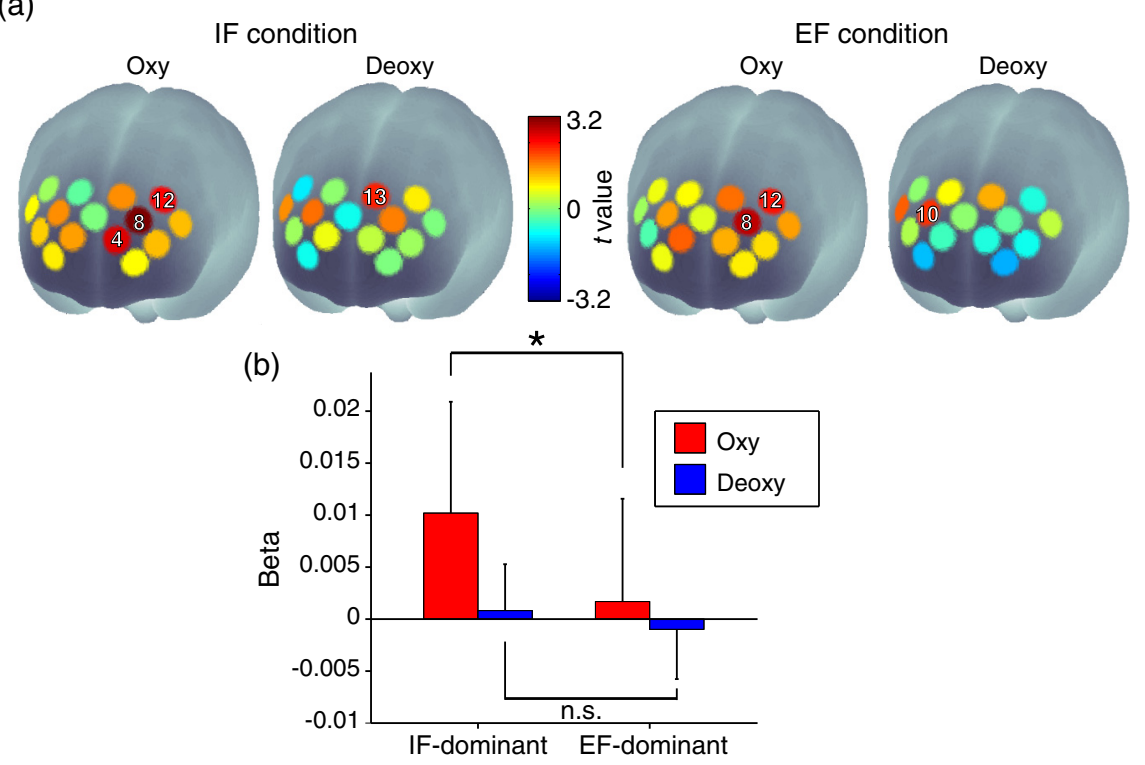

Fig. 6 Spatial configuration in the prefrontal area reflecting individual optimal attentional strategy. (a) Only the beta value of oxy- $\mathrm{Hb}$ signal in ch.8 under the IF condition showed a significant difference between IF- and EF-dominant subgroups. (b) Beta values in ch. 8 under the IF condition. The positive and negative values indicate an increase and a decrease in oxy- $\mathrm{Hb} /$ deoxy- $\mathrm{Hb}$ signals, respectively. The beta value of oxy-Hb signal in the IF-dominant subgroup was significantly larger than that in the EF-dominant subgroup ( $p=0.0016$; $t$-test, FDR-corrected). ${ }^{*} p<0.05$.

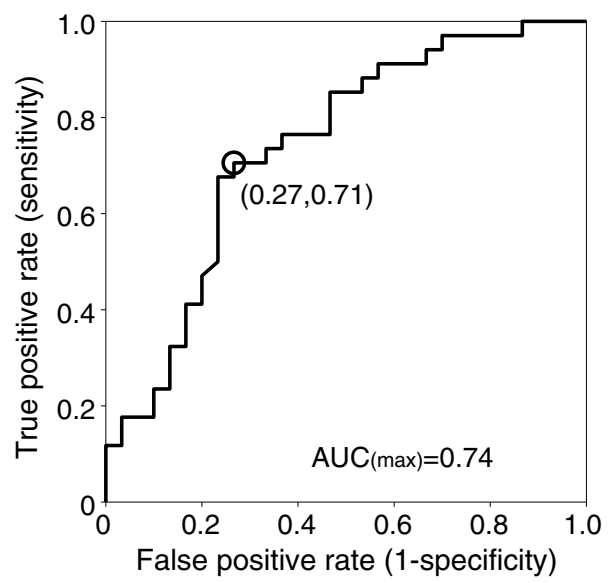

Fig. 7 ROC curve based on the beta value of left prefrontal activity (ch.8). The open circle indicates the optimal sensitivity and specificity when we applied a cut-off value of the highest AUC.

\section{Discussion}

Consistent with our previous studies, ${ }^{10,11}$ we found individual differences in the optimal attentional strategy for improving motor performance in healthy and clinical populations. In contrast to previous studies concluding that EF is generally superior, we replicated our previous finding that the EF strategy does not lead to better motor performance in a substantial subset of participants. Furthermore, in this study, we revealed the common neural basis of individual optimal attentional strategy. Contrary to previous neuroimaging studies demonstrating that motorrelated areas are involved in the effect of focus of attention, ${ }^{12,13,43}$ we found that the oxy-Hb signal change in the left FPC and left dorsolateral prefrontal cortex (DLPFC) during the IF strategy reflected individual motor performance. This finding supports our hypothesis that the prefrontal area is an important region encoding individual optimal attentional strategy. Based on this finding, we further developed a classification method for IF-dominant and EF-dominant individuals based on task-related neural activity in the left FPC and DLPFC. We propose that cognitive processing of internal body information in the left FPC and DLPFC is a critical determinant of individual optimal attentional strategy for motor tasks.

We presume that the left FPC and DLPFC are both important areas underlying individual optimal attentional strategy in healthy and clinical populations during motor tasks. The prefrontal cortex contributes to multiple cognitive functions such as attentional control and working memory ${ }^{44}$ as well as processing attention to action through connectivity with the premotor cortex. ${ }^{45}$ This functional link between the prefrontal cortex and motor-related areas is consistent with current findings that individual activity in the FPC and DLPFC is linked to motor performance. Notably, we found individual differences in left FPC and DLPFC activity patterns only under the IF condition. Specifically, individuals with IF-dominance showed stronger task-related activation in these areas than those with EFdominance. These findings indicate that the individual ability of the FPC and DLPFC to process internal sensorimotor information-not information from the external environment (such as visual stimuli) - determines the optimal attentional strategy during motor tasks. In fact, several previous neuroimaging studies support that the left prefrontal cortex is relevant to sensory processing of internal body information. For example, during a tactile discrimination task, left DLPFC activity increased as a function of the relative frequency difference in correct decision trials. ${ }^{14}$ The authors concluded that the left DLPFC encodes stimulus representations that underlie veridical tactile decisions. ${ }^{14}$ Another study found a significant difference in left DLPFC activity among different somatosensory memory loads, ${ }^{46}$ and Kaas et al. reported that left FPC activity was associated with 
working memory required to maintain representations of haptic information and integrate spatial and motor components. ${ }^{15}$ These previous reports regarding prefrontal areas in cognitive processing relevant to internal body state support our suggestion that the left prefrontal region is a critical regulator of individual optimality of IF. Alternatively, the prefrontal activity patterns observed in this study could reflect individual sensitivity to sensory inputs of internal body information. In other words, we cannot fully reject the possibility that individual characteristics of sensory processing, rather than individual cognitive ability, affected prefrontal activity. Nevertheless, it is certain that the individual ability for processing internal body information based on the prefrontal cortex can characterize the optimal attentional strategy during motor tasks.

Cognitive load theory offers an additional interpretation of the relationship between FPC and DLPFC activity and motor performance. ${ }^{18-20}$ If FPC and DLPFC strongly reflect the intrinsic load in the current task, we could expect that relatively higher activity in these regions is observed under a nonoptimal attentional condition. However, contrary to this expectation, individuals who were good at directing their attention to body movements (i.e., the IF-dominant subgroup) showed higher activity under the IF condition. Alternatively, the current findings suggest that relatively higher activity in the FPC and DLPFC under the IF condition reflects germane load, contributing to improved motor performance. ${ }^{19}$ In other words, encouraging the assignment of a cognitive resource to the germane load might be a fundamental benefit of an individual's optimal attentional strategy during motor tasks.

The prefrontal cortex is also reportedly associated with the processing of external information. For example, the prefrontal cortex encodes spatial object configuration for visuospatial memory ${ }^{47}$ and evaluates specific visual memory characteristics. ${ }^{48}$ Based on these previous reports, we would expect distinct neural activation patterns between EF- and IF-dominant individuals under not only the IF condition but also the EF condition. However, in this study, we observed the distinct neural activation only under the IF condition. This discrepancy could be explained by task difficulty: when a motor task is too easy, the effects of EF strategy are not expected. ${ }^{11,49,50}$ Hence, the simple motor task in the current fNIRS study, which required that participants make simple cyclical hand movements, may not have been difficult enough to engage substantial attentional resources under the EF condition. Thus, the stronger effects on motor task under the IF condition imply that IF would be more helpful for assessing individual optimal attentional strategies.

Left prefrontal activity during the simple motor task has the potential to be an accurate objective biomarker to predict individual optimal attentional strategies that lead to better motor performance. Specifically, an individual showing more intense activity in the left prefrontal cortex could better prevent motor performance decline by applying the IF strategy in a novel environment. Given that the left prefrontal cortex is one of the critical regions for processing somatosensory information, ${ }^{14}$ it is reasonable to expect that activity in this area will distinguish the individual optimal attentional strategy during motor tasks. Although the sample size in this study was insufficient to investigate classification analysis, the optimal cut-off value calculated using ROC analysis successfully classified individual differences in all participant groups, which supports that the left prefrontal area is the common neural basis reflecting the optimal attentional strategy in both healthy and clinical populations.
Of note, symptomatic states such as lesion site, lesion side, severity, and FMA score were heterogeneous in our clinical population. A previous study reported neuroplastic changes in network connectivity among prefrontal and motor-related areas following stroke. ${ }^{51}$ Nevertheless, the stroke group showed equivalent classification accuracy as the young and elderly groups. This result suggests that the prefrontal activity-based classification has sufficient robustness that we do not need to consider a patient's lesion side or handedness. Although we previously tried to classify the optimal attentional strategy using self-report questionnaires on motor imagery, ${ }^{10,11}$ assessment using questionnaires is simple but highly subjective. The current fNIRS approach is useful to overcome such subjectivity.

The individual differences in optimal attentional strategy during motor tasks require a framework alternative to the constrained-action hypothesis. ${ }^{4}$ The traditional hypothesis is that applying the EF strategy leads to greater motor automaticity and enhances motor improvement. However, the current findings suggest that attentional focus on an individual familiar sensory modality (i.e., internal body information such as tactile/somatosensory or external environment information such as visual stimuli) can also contribute to better motor performance. The major difference between our new framework and the constrained-action hypothesis is that the positive effect of attentional focus on motor control can be acquired not only under the EF strategy but also under the IF strategy. Notably, we might benefit from an individual optimal attentional strategy based on familiar sensory modalities in a specific aspect of motor learning. The motor learning process is modeled with a two-time scale function combined with a transient change (i.e., fast time scale) and a persistent change (i.e., slow time scale) ${ }^{52}$ In our current study, participants exhibited a relatively small motor performance decline from the initial trials to the relearning phase under the individual optimal attentional condition, suggesting that the optimal strategy enhances the slow time scale process links to consistently better motor performance.

Finally, we need to consider that fNIRS signals are affected not only by the cerebral blood flow associated with local neural activity but also by the extracerebral physiological factors such as blood pressure or blood $\mathrm{CO}_{2}$ concentration. ${ }^{53}$ In addition, motor tasks can elicit a global component in the oxy-Hb signals. $^{54,55}$ These factors to be excluded can yield a higher false-positive rate or false-negative rate in the analysis for fNIRS studies. ${ }^{56}$ Even in the current study, all extracerebral physiological factors pointed out in the previous studies might be not removed. However, to detect the cerebral blood flow originated from neural activity, we applied multidistance $\mathrm{ICA}^{30}$ and estimated neural activity-based on GLM analysis. ${ }^{37,38}$ Furthermore, we succeeded in confirming the typical oxy/deoxy hemodynamic patterns. In particular, the asymmetric responses between the oxy-Hb signals and the deoxy-Hb signals observed in the current fNIRS task (larger activities in the oxy-Hb signals compared with the deoxy-Hb signals) agree with a previous report that the oxy-Hb signals have greater sensitivity to change in cerebral blood flow and higher signal-to-noise ratio than the deoxy-Hb signals. ${ }^{57}$ Thus, we can assume that the current results based on the beta values from GLM analysis were correctly detected as task-related neural activities. Of note, a previous study reported that the amplitude of the deoxy- $\mathrm{Hb}$ signal is approximately half of the amplitude of the oxy-Hb signal, ${ }^{58}$ however, the deoxy-Hb signal in this study was relatively small compared with those in the previous study. The small responses 
in the deoxy-Hb signal may be caused by the multidistance ICA to remove the global component.

A major limitation of this study is that the motor task used was rather short-term and simple compared with tasks used in clinical rehabilitation or sports training. Although our findings imply that left FPC and DLPFC activities are associated with an individual's attentional control ability, it is necessary to verify whether this holds true for long-term or complex motor tasks that are more reflective of daily activities. We have not directly investigated this point; however, both the current study and our previous work ${ }^{10,11}$ show individual differences in optimal attentional strategy. The consistency in the individual differences among different visuomotor tasks implies that individual differences in optimal attentional strategy are not restricted to one simple task and suggests that such differences are broadly applicable to rehabilitation and training applications. Second, the clinical population comprised only stroke patients with mild or moderate paralysis. Further research is needed to clarify the relationship between individual optimal attentional strategy and degree of severity. In addition, we need further exploration of whether other individual factors such as gender in each group affect the prefrontal activity depending on the optimal attentional strategy.

In conclusion, directing attention to body movements was found to differentially modulate prefrontal cortex activities, especially left FPC and DFLPC activities. These regions may be critical for determining an individual's optimal attentional strategy for improving motor performance. Optimal attentional strategy classification based on differential prefrontal activity could contribute to the development of personalized rehabilitation and sports training programs.

\section{Disclosures}

All authors declare no conflicts of interest.

\section{Acknowledgments}

We thank laboratory members for their support and encouragement. We also thank the occupational therapists at the rehabilitation center of Jichi Medical University Hospital for conducting FMA motor impairment assessments. This research was supported by JSPS KAKENHI, Grant Nos. 15K16366 (to T.S.) and 25871228 (to M.H.).

\section{References}

1. G. Wulf, C. Shea, and R. Lewthwaite, "Motor skill learning and performance: a review of influential factors," Med. Educ. 44(1), 75-84 (2010).

2. S. Y.-C. Peh, J. Y. Chow, and K. Davids, "Focus of attention and its impact on movement behaviour," J. Sci. Med. Sport 14(1), 70-78 (2011).

3. G. Wulf, "Attentional focus and motor learning: a review of 15 years," Int. Rev. Sport Exercise Psychol. 6(1), 77-104 (2013).

4. G. Wulf, N. McNevin, and C. H. Shea, "The automaticity of complex motor skill learning as a function of attentional focus," $Q$. J. Exp. Psychol. A 54(4), 1143-1154 (2001).

5. N. H. McNevin, C. H. Shea, and G. Wulf, "Increasing the distance of an external focus of attention enhances learning," Psychol. Res. 67(1), 22-29 (2003).

6. T. Zachry et al., "Increased movement accuracy and reduced EMG activity as the result of adopting an external focus of attention," Brain Res. Bull. 67(4), 304-309 (2005).

7. N. Perkins-Ceccato, S. R. Passmore, and T. D. Lee, "Effects of focus of attention depend on golfers' skill," J. Sports Sci. 21(8), 593-600 (2003).
8. M. Emanuel, T. Jarus, and O. Bart, "Effect of focus of attention and age on motor acquisition, retention, and transfer: a randomized trial," Phys. Ther. 88(2), 251-260 (2008).

9. E. C. Kal et al., "Stay focused! The effects of internal and external focus of attention on movement automaticity in patients with stroke," PLoS One 10(8), e0136917 (2015).

10. T. Sakurada, M. Hirai, and E. Watanabe, "Optimization of a motor learning attention-directing strategy based on an individual's motor imagery ability," Exp. Brain Res. 234(1), 301-311 (2016).

11. T. Sakurada et al., "Improved motor performance in patients with acute stroke using the optimal individual attentional strategy," Sci. Rep. 7, 40592 (2017).

12. K. Zentgraf et al., "Neural correlates of attentional focusing during finger movements: a fMRI study," J. Motor Behav. 41(6), 535-541 (2009).

13. K. M. Zimmermann et al., "Neural correlates of switching attentional focus during finger movements: an fMRI study," Front. Psychol. 3, 555 (2012).

14. B. Pleger et al., "Neural coding of tactile decisions in the human prefrontal cortex," J. Neurosci. 26(48), 12596-12601 (2006).

15. A. L. Kaas, H. Van Mier, and R. Goebel, "The neural correlates of human working memory for haptically explored object orientations," Cereb. Cortex 17(7), 1637-1649 (2007).

16. J. Sweller, "Cognitive load during problem solving: effects on learning," Cognit. Sci. 12(2), 257-285 (1988).

17. J. Sweller, J. J. G. van Merrienboer, and F. G. W. C. Paas, "Cognitive architecture and instructional design," Educ. Psychol. Rev. 10(3), 251-296 (1998).

18. E. Koechlin and A. Hyafil, "Anterior prefrontal function and the limits of human decision-making," Science 318(5850), 594-598 (2007).

19. P. A. Shewokis et al., "Acquisition, retention and transfer of simulated laparoscopic tasks using fNIR and a contextual interference paradigm," Am. J. Surg. 213(2), 336-345 (2017).

20. R. R. Whelan, "Neuroimaging of cognitive load in instructional multimedia," Educ. Res. Rev. 2(1), 1-12 (2007).

21. G. Allali et al., "The neural basis of age-related changes in motor imagery of gait: an fMRI study," J. Gerontol. A 69(11), 1389-1398 (2014).

22. M. E. Berryhill and K. T. Jones, "tDCS selectively improves working memory in older adults with more education," Neurosci. Lett. 521(2), 148-151 (2012).

23. M. F. Folstein, S. E. Folstein, and P. R. McHugh, "'Mini-mental state.' A practical method for grading the cognitive state of patients for the clinician," J. Psychiatr. Res. 12(3), 189-198 (1975).

24. R. C. Oldfield, "The assessment and analysis of handedness: the Edinburgh inventory," Neuropsychologia 9(1), 97-113 (1971).

25. A. R. Fugl-Meyer et al., "The post-stroke hemiplegic patient. 1. A method for evaluation of physical performance," Scand. J. Rehabil. Med. 7(1), 13-31 (1975).

26. "Cogent I Wellcome Laboratory of Neurobiology," http://www.vislab .ucl.ac.uk/cogent.php (accessed 8 June 2019).

27. D. F. Hultsch, S. W. S. MacDonald, and R. A. Dixon, "Variability in reaction time performance of younger and older adults," J. Gerontol. Ser. B 57(2), P101-P115 (2002).

28. R. Ratcliff, "Methods for dealing with reaction time outliers," Psychol. Bull. 114(3), 510-532 (1993).

29. E. Dayan and L. G. Cohen, "Neuroplasticity subserving motor skill learning," Neuron 72(3), 443-454 (2011).

30. T. Funane et al., "Quantitative evaluation of deep and shallow tissue layers' contribution to fNIRS signal using multi-distance optodes and independent component analysis," Neuroimage 85, 150-165 (2014).

31. C. B. Akgül, A. Akin, and B. Sankur, "Extraction of cognitive activityrelated waveforms from functional near-infrared spectroscopy signals," Med. Biol. Eng. Comput. 44(11), 945-958 (2006).

32. R. Hirosaka et al., "Noisy time-delayed decorrelation and its application to extraction of neural activity from single optical recordings in guinea pigs," Phys. D Nonlinear Phenom. 194(3-4), 320-332 (2004).

33. G. Morren et al., "Detection of fast neuronal signals in the motor cortex from functional near infrared spectroscopy measurements using independent component analysis," Med. Biol. Eng. Comput. 42(1), 92-99 (2004). 
34. S. Kohno et al., "Removal of the skin blood flow artifact in functional near-infrared spectroscopic imaging data through independent component analysis," J. Biomed. Opt. 12(6), 062111 (2007).

35. A. K. Singh et al., "Spatial registration of multichannel multi-subject fNIRS data to MNI space without MRI," Neuroimage 27(4), 842-851 (2005).

36. "NITRC: Homer2: Tool/Resource Info," NeuroImaging Tools \& Resources Collaboratory, https://www.nitrc.org/projects/homer2/ (accessed 8 June 2019).

37. K. J. Friston, P. Jezzard, and R. Turner, "Analysis of functional MRI time-series," Hum. Brain Mapp. 1(2), 153-171 (1994).

38. K. J. Friston et al., "Statistical parametric maps in functional imaging: a general linear approach," Hum. Brain Mapp. 2(4), 189-210 (1995).

39. M. L. Schroeter et al., "Towards a standard analysis for functional nearinfrared imaging," Neuroimage 21(1), 283-290 (2004).

40. M. M. Plichta et al., "Model-based analysis of rapid event-related functional near-infrared spectroscopy (NIRS) data: a parametric validation study," Neuroimage 35(2), 625-634 (2007).

41. Y. Benjamini and Y. Hochberg, "Controlling the false discovery rate: a practical and powerful approach to multiple testing," J. R. Stat. Soc. Ser. B 57(1), 289-300 (1995).

42. A. K. Singh and I. Dan, "Exploring the false discovery rate in multichannel NIRS," Neuroimage 33(2), 542-549 (2006).

43. Y. A. Kuhn et al., "Adopting an external focus of attention alters intracortical inhibition within the primary motor cortex," Acta Physiol. 220(2), 289-299 (2017).

44. K. Kollndorfer et al., "Altered likelihood of brain activation in attention and working memory networks in patients with multiple sclerosis: an ALE meta-analysis," Neurosci. Biobehav. Rev. 37(10), 2699-2708 (2013).

45. J. Rowe et al., "Attention to action: specific modulation of corticocortical interactions in humans," Neuroimage 17(2), 988-998 (2002).

46. N. Savini et al., "Working memory of somatosensory stimuli: an fMRI study," Int. J. Psychophysiol. 86(3), 220-228 (2012).

47. S. D. Slotnick and L. R. Moo, "Prefrontal cortex hemispheric specialization for categorical and coordinate visual spatial memory," Neuropsychologia 44(9), 1560-1568 (2006).

48. C. Ranganath, M. K. Johnson, and M. D'Esposito, “Left anterior prefrontal activation increases with demands to recall specific perceptual information," J. Neurosci. 20(108), RC108 (2000).

49. M. Landers et al., "An external focus of attention attenuates balance impairment in patients with Parkinson's disease who have a fall history," Physiotherapy 91(3), 152-158 (2005).

50. G. Wulf, T. Töllner, and C. H. Shea, "Attentional focus effects as a function of task difficulty," Res. Q. Exercise Sport 78(4), 257-264 (2007).

51. N. Sharma, J.-C. Baron, and J. B. Rowe, "Motor imagery after stroke: relating outcome to motor network connectivity," Ann. Neurol. 66(5), 604-616 (2009).

52. M. E. Joseph, A. C. King, and K. M. Newell, "Task difficulty and the time scales of warm-up and motor learning," J. Motor Behav. 45(3), 231-238 (2013).

53. M. Caldwell et al., "Modelling confounding effects from extracerebral contamination and systemic factors on functional near-infrared spectroscopy," Neuroimage 143, 91-105 (2016).

54. X. Zhang, J. A. Noah, and J. Hirsch, "Separation of the global and local components in functional near-infrared spectroscopy signals using principal component spatial filtering," Neurophotonics 3(1), 015004 (2016).

55. D. A. Boas, A. M. Dale, and M. A. Franceschini, "Diffuse optical imaging of brain activation: approaches to optimizing image sensitivity, resolution, and accuracy," Neuroimage 23, S275-S288 (2004).
56. I. Tachtsidis and F. Scholkmann, "False positives and false negatives in functional near-infrared spectroscopy: issues, challenges, and the way forward," Neurophotonics 3(3), 031405 (2016).

57. G. Strangman et al., "A quantitative comparison of simultaneous BOLD fMRI and NIRS recordings during functional brain activation," Neuroimage 17(2), 719-731 (2002).

58. V. Toronov et al., "Investigation of human brain hemodynamics by simultaneous near-infrared spectroscopy and functional magnetic resonance imaging," Med. Phys. 28(4), 521-527 (2001).

Takeshi Sakurada received his $\mathrm{PhD}$ from Tokyo Institute of Technology, Japan, in 2010. He is currently an assistant professor at the College of Science and Engineering, Ritsumeikan University, Japan. His research interests include motor learning in healthy populations and neurorehabilitation for stroke patients with motor disabilities.

Aya Goto is a master's course student at Shibaura Institute of Technology, Japan. Her research is focused on developing tailormade rehabilitation programs based on individual differences in the neural motor system.

Masayuki Tetsuka received his medical degree from Aichi Medical University, Japan, in 2009. He is currently an assistant professor in the Department of Neurosurgery, Jichi Medical University, and is also a doctoral course student at Jichi Medical University. His research is focused on neurorehabilitation based on a neurofeedback system using fNIRS.

Takeshi Nakajima received his medical degree from Akita University, Japan, in 1997, and his PhD from Tohoku University, Japan, in 2003. $\mathrm{He}$ is a lecturer in the Department of Neurosurgery, Jichi Medical University, and is concurrently a lecturer at the Rehabilitation Center, Jichi Medical University Hospital. His research is focused on neurological surgery for epilepsy and deep brain stimulation for Parkinson's disease.

Mitsuya Morita received his medical degree from Jichi Medical University, Japan, in 1986, and his PhD from Jichi Medical University, Japan, in 1995. He is an associate professor in the Division of Neurology of the Department of Internal Medicine, Jichi Medical University, and is concurrently an associate professor at the Rehabilitation Center at Jichi Medical University Hospital. His research is focused on motor neuron disease.

Shin-ichiroh Yamamoto received his PhD from the University of Tokyo, Japan, in 2000. He is currently a professor at the College of Systems Engineering and Science, Shibaura Institute of Technology, Japan. His main research interests include neuroengineering for postural control and gait.

Masahiro Hirai received his PhD from the University of Tokyo, Japan, in 2005. He is currently an associate professor at the Functional Brain Science Section, Jichi Medical University, Japan. His research is focused on the neural mechanisms underlying social cognition in typical and atypical development.

Kensuke Kawai received his medical degree from the University of Tokyo, Japan, in 1987, and his PhD from the University of Tokyo, Japan, in 1995. He is a professor in the Department of Neurosurgery at Jichi Medical University. His main research interests include evaluation of surgical treatment and development of innovative treatments for epilepsy. 